\title{
Fixierung von Hauttransplantaten mit Vakuumversiegelung: Eine sinnvolle Option?
}

\author{
Negative pressure wound therapy for skin graft fixation: \\ A reasonable option?
}

\author{
Autor \\ Markus Meissner \\ Institut \\ Klinik für Dermatologie, Venerologie und Allergologie, \\ Johann Wolfgang Goethe-Universität, Frankfurt am Main, \\ Deutschland \\ Schlüsselwörter \\ Vakuumversiegelung, Spalthauttransplantat, chronische \\ Wunde, randomisierte kontrolliert Studie
}

Key words

Negative pressure wound therapy, split thickness skin graft, chronic wound, randomized controlled study

eingereicht 19.07.2019

akzeptiert 22.07.2019

Bibliografie

DOI https://doi.org/10.1055/a-0986-0452

Online-Publikation: 15.08.2019

Phlebologie 2019; 48: 311-316

(c) Georg Thieme Verlag KG Stuttgart · New York ISSN 0939-978X

Korrespondenzadresse

Prof Dr. Dr. Markus Meissner

Klinik für Dermatologie, Venerologie und Allergologie

Klinikum der J. W. Goethe-Universität

Theodor-Stern-Kai 7

60590 Frankfurt am Main

Tel.: + 4969 6301-6845

Fax: + 4969 6301-3804

E-Mail: markus.meissner@kgu.de $\oplus$

Englische Version unter:

https://doi.org/10.1055/a-0986-0452

\section{ZUSAMMENFASSUNG}

Die Vakuumversiegelung (VS) ist eine seit circa 20 Jahren in der klassischen Wundversorgung nicht mehr wegzudenkende Therapieoption. Insbesondere im Bereich der Wundgrundkonditionierung von chronischen Wunden nimmt sie einen wichtigen Stellenwert ein. In den letzten Jahren wird die Vakuumversiegelung auch zur Fixierung und dem postoperativen Wundmanagement nach Hauttransplantation immer häufiger eingesetzt. Die vorliegende Übersichtsarbeit beschäftigt sich mit den Daten und Evidenzen eines solche Vorgehens und zeigt Möglichkeiten der Nutzung auf.

\section{ABSTRACT}

Negative pressure wound therapy (NPWT) is meanwhile since about 20 years a standard procedure in classical wound care. Especially in wound ground conditioning of chronic wounds NPWT has an important status. In the last years, NPWT was more and more used in the fixation and postoperative wound management of skin grafts. The current review addresses the available data and evidence and demonstrates the possible use of the procedure.

\section{Einleitung}

Die Versorgung von komplexen Hautwunden, seien sie akut oder auch chronisch, sind seit jeher eine ärztliche Herausforderung. Neben der Ursachenbehandlung ist die stadiengerechte Wundversorgung ein zentraler Schwerpunkt des modernen Wundmanagements [17]. Die Anwendung von Spalthauttransplantaten (SHT) gehören zu den Standardprozeduren in der Versorgung und ermöglicht einen schnellen und effektiven Verschluss. Für die Einheilung des Transplantats sind in den ersten 3-5 Tagen die Verhinderung von Scherkräften, von subtransplantaren Seromen und Hämatomen sowie von Infektionen essentiell. Die Fixierung wird klassischerweise durch einen Überknüpfungsverband mit Wunddistanzgitter durchgeführt. Hierbei ist die Druckverteilung meistens ungleichmäßig und die Fähigkeit des Verbandes Wundsekrete auf- 
zunehmen ist insbesondere bei größeren Wunden eingeschränkt [6]. Auch die Anpassung an sehr große, unebene oder bewegliche Wundflächen kann sich mit dem klassischen Verband schwierig gestalten und führt zu geringeren Einheilungsraten [23, 25]. Die postoperative Vakuumversiegelung scheint die Nachteile des klassischen Verbandes zu umgehen. Hierbei spielen die feste, gleichmäßige und bewegungsstabile Fixierung auch in Problemregionen, ein effektives Exsudat-Management und ein feuchtes Wundmilieu sicher eine entscheidende Rolle [23]. Aus diesen Gründen wird die postoperative Vakuumversiegelung nach Hauttransplantationen mittlerweile immer häufiger angewendet. Trotz der subjektiv empfundenen verbesserten Einheilungsraten ist die Evidenz zu diesem Verfahren jedoch noch gering.

\section{Wirkweise der Vakuumversiegelung}

1997 wurde von Argenta und Morykwas die Vakuumversiegelung entwickelt, die mittlerweile als standardisierte kommerziell erhältliche Systeme zur Verfügung stehen [1]. Die Wirkweise ist noch nicht bis in alle Einzelheiten geklärt und weiterhin ein Feld der Grundlagenwissenschaft. Zwei wichtige Grundprinzipien scheinen eine essentielle Rolle zu spielen. Auf der einen Seite die Makrodeformation der Wunde durch zentripetal gerichteten Zug auf die Wundränder, die auch im Zusammenhang mit dem angelegten Sog zu einer Ödemreduktion führt. In Arbeiten von Morykwas et al. konnte gezeigt werden, dass hierdurch eine vermehrte Durchblutung des Gewebes induziert wird [19]. Kairinos et al. und Wackenfors et al. zeigen hingegen in ihren Arbeiten eine passager verminderte Durchblutung, die aber in der Folge zur vermehrten Angiogenese und Zellproliferation führte [10, 24]. Hier wird eine Hypoxie vermittelte Aktivierung des HIF1 $\alpha$-Signalweges angenommen. Neben der Makrodeformation spielt wahrscheinlich die Wechselwirkung zwischen dem offenporigen Polyurethan-Schwamm und der Wunde eine entscheidende Rolle. Diese Mikrodeformation führt zu einem „stretching“ der Zellverbände, was nachweislich zu einer erhöhten Proliferationsrate der Zellen führt [5]. Zusätzlich kommt es zur vermehrten Bildung von wichtigen granulationsfördernden Zytokinen wie zum Beispiel VEGF, bFGF oder II-8 [10, 16]. Auch die Kollagenproduktion und die Migration der Zellen wird induziert [9]. Neuste Daten zeigen, dass die VS auch zur Anreicherung von zirkulierenden Fibrozyten in den Wunden führt [4]. Diese Arbeit zeigt damit, dass auch eine systemische Beeinflussung durch die VS induziert werden kann.

Weitere essentielle Faktoren, die zur optimalen Wundumgebung beitragen, sind das feuchte Wundmilieu bei gleichzeitiger Entfernung von überschüssigen Wundsekreten, die häufig auch wundheilungsinhibierende Faktoren wie beispielsweise Matrix-Metalloproteinasen enthalten sowie eine konstante Wundtemperatur. Zusätzlich wird von einigen Autoren eine Reduktion der Keimbelastung der Wunde postuliert. So konnte Wang et al. zeigen, dass es unter VS zu einer relevanten Reduktion von Pseudomonas aeroginosa kommt [7]. Zu ähnlichen Ergebnissen kamen Li et al. im Hinblick auf Staphylococcus aureus [14].

\section{Vorgehen bei Vakuumversiegelung nach Spalthauttransplantation}

Die erste Studie über die Anwendung der VS von Spalthauttransplantaten bei über 100 chronische Wunden stammt von Schneider et al. aus dem Jahre 1998 [22]. In der Arbeit wurde die Methode und das Vorgehen erstmalig ausführlich beschrieben. Genauere Analysen der Patientendaten wurden nicht durchgeführt. Es wurde lediglich berichtet, dass es nur bei zwei Patienten zu einem Verlust des Transplantates gekommen sei. Das in der Arbeit beschriebene Vorgehen wird mit individuellen Abwandlungen auch heute noch so durchgeführt ( $\mathbf{A b b}$. 1a $\rightarrow$ Abb. 1e). Insbesondere auch an schwierigen Lokalisationen (z. B. im Bereich der Gelenke, genital/inguinal/axillär Region) kann die VS die Fixierung des Spalthauttransplantates wesentlich vereinfachen und auch die Wundfläche sicher vor Kontaminationen schützen ( $\vee$ Abb. 2a- $\mathbf{A b b}$. 2f). Die verwendeten Soge liegen in den verschiedenen Arbeiten zwischen $75-125 \mathrm{mmHg}$. Ein dünnes Silikon Wunddistanzgitter ist zwischen Schwamm und Transplantat zu empfehlen ( $>$ Abb. 2d). Im Durchschnitt verbleibt die Vakuumversiegelung über 5 Tage (3-7 Tage), ein Wechsel des funktionierenden Systems ist in dieser Zeit nicht notwendig. Wundinfekte sind auf Grund der guten Sichtbarkeit der Wundränder sofort zu erkennen. In diesem Fall muss die Vakuumversiegelung beendet und auf klassische Verbände gewechselt werden.

\section{Kontrolliert randomisierte Studien}

Zur vorgestellten Methode gibt leider nur sehr wenige Studien. Der Großteil ist nicht kontrolliert und randomisiert und die meisten sind nur retrospektiv. Trotzdem gibt es einige Arbeiten, die sehr gute Hinweise für eine Wirksamkeit der Therapie geben.

Die erste randomisiert kontrollierte Studie, die den Vergleich zwischen VS und klassischem Überknüpfungsverband untersuchte war von Moisidis et al. aus dem Jahr 2004 [18]. Insgesamt wurden 20 Patienten eingeschlossen und es wurde ein intraindividueller Vergleich gezogen, indem die eine Hälfte des Defekts klassisch verbunden wurde, die andere Hälfte mittels Vakuumversiegelung. Insgesamt unterschieden sich die Defekte nicht in der quantitativen Einheilungsrate aber in der Qualität des eingeheilten Transplantats, bei der die VS ein signifikant besseres Outcome hatte. Hierbei handelt es sich zwar um ein interessantes Design, aber es ist fraglich, ob die beiden Wundanteile wirklich unabhängig voneinander betrachtet werden können, da die VS nachweislich die Mikrozirkulation auch noch in einem Abstand von 2-3 Zentimetern beeinflusst und auch systemische Faktoren wie die Mobilisierung zirkulierender Fibrozyten unter VS eine Rolle für die Wundheilung spielen.

Die Studie von Llanos et al. ist die bisher beste und umfangreichste prospektive randomisierte und kontrollierte Studie [15]. Insgesamt wurden 60 Patienten in zwei Gruppen, eine VS und eine Kontrollgruppe mit alleinigem Verband ohne Sog untersucht. Die Autoren konnten zeigen, dass in der VS-Gruppe signifikant weniger Transplantatverlust vorlag als in der Kontrollgruppe. Zusätzlich konnte die Zeit von Transplantation zur Entlassung von im Mittel 12 auf 8 Tage reduziert werden. In der Kontrollgruppe benötigen 12 Patienten (40\%) eine erneute Transplantation in der VS-Gruppe waren es nur 5 Patienten (17\%). Interessanterweise zeigte sich 

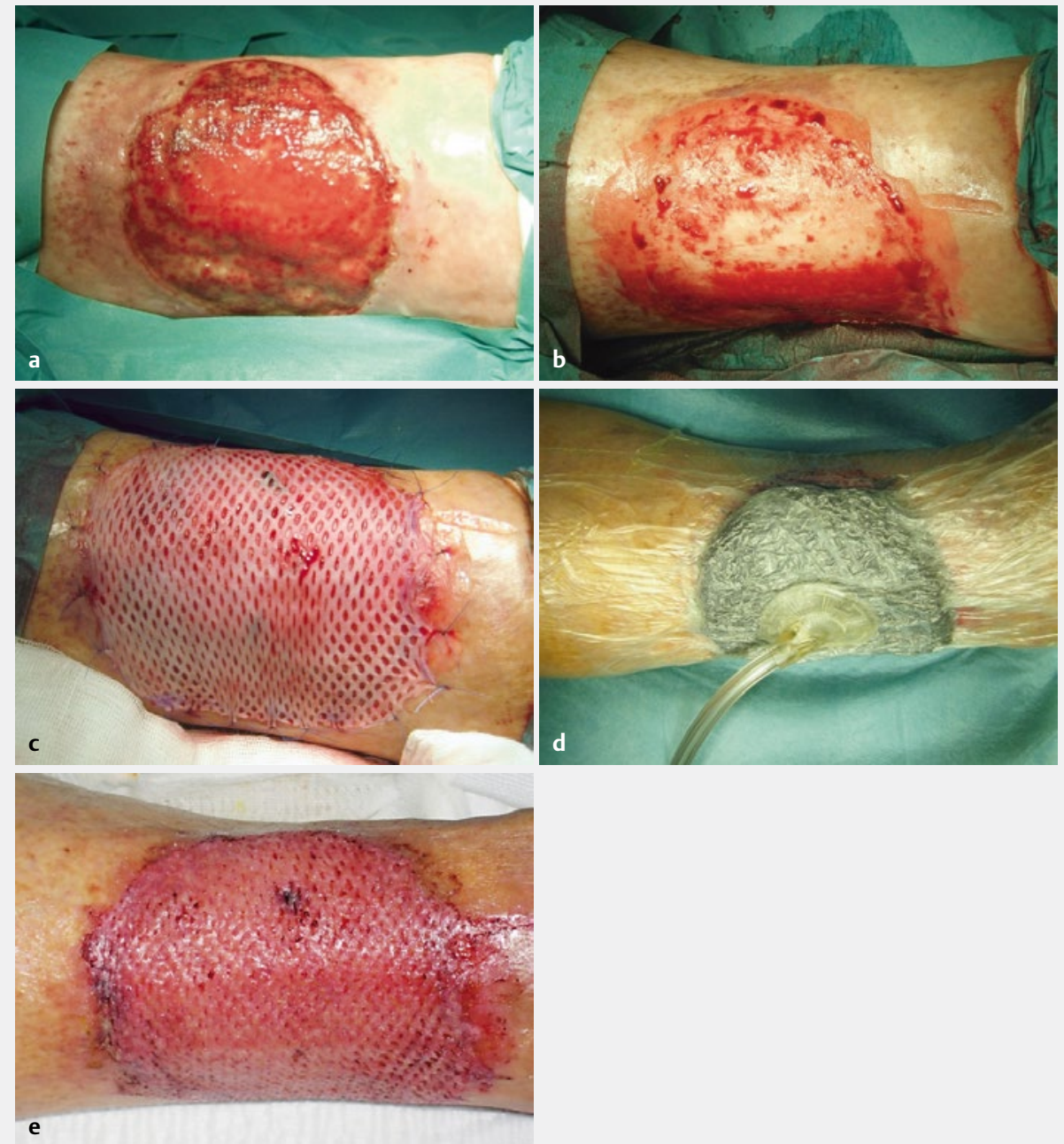

Abb. 1 Vakuumversiegelung eines Spalthauttransplantats nach Ulcus-Shaving im Bereich des Unterschenkels. a Ulcus cruris präoperativ; b Zustand nach Ulcus Shaving; c mit einigen Fixationsnähten befestigtes Spalthauttransplantat; d Fixierung des Transplantats mittels Vakuumversiegelung; e Zustand nach Abnahme der Vakuumversiegelung nach 5 Tagen.

bei der Kontrollgruppe ein direkter Zusammenhang zwischen der Größe des transplantierten Areals und der Wahrscheinlichkeit eines Transplantatverlusts, wohingegen in der VS-Gruppe ein solcher Zusammenhang nicht vorlag. Dies mag an der wesentlich besseren und über die Wundfläche gleichmäßigeren Fixierung durch die Vakuumversiegelung liegen, was bei großen Wundflächen mit dem klassischen Verband nicht so gut gewährleistet werden kann.

Zu ähnlichen Daten wie die Studie von Llanos kamen Petkar et al. in einer ebenfalls randomisierten prospektiven kontrollierten Studie, die bei Patienten mit Brandverletzungen in der VS-Gruppe 21 Patienten und in der Kontrollgruppe 19 Patienten einschlossen [20]. Auch hier kam es zu einer signifikant besseren Einheilungsrate $97 \%$ versus $88 \%$ (VS versus Kontrolle) und die Zeit bis zur kompletten Verbandabnahme konnte von 11 Tagen in der Kontrollgruppe auf 8 Tage in der VS Gruppe reduziert werden.

Eine neuere RCT von Hsiao et al. mit insgesamt 28 Patienten (14 Patienten in jeder Gruppe) zeigte zwar keinen signifikanten Unterschied was die Einheilungsrate angeht, aber die Patienten litten unter wesentlich weniger Schmerzen (im Mittel bis zu 4 Punkte in der visuellen Analogskala) unter der Vakuumversiegelung [8]. Zusätzlich waren die Patienten im Vergleich zum Standardverband signifikant zufriedener mit der Behandlung und dem Ergebnis. Hierzu tragen sicher die geringeren Schmerzen und die wesentlich höhere Mobilität, die unter einer VS möglich ist, bei. Insbesondere die verbesserte Mobilität der Patienten durch die Vakuumversiegelung sollte nicht unterschätzt werden, da hierdurch nicht nur die Patientenzufriedenheit und Selbstständigkeit erhalten werden kann, sondern gleichzeitig auch eine aktive Thromboseprophylaxe durchgeführt wird.

Eine weitere aktuelle neuseeländische Studie hat den Einfluss der VS auf die erhöhte Mobilität und damit die wesentlich frühzeitigere Entlassbarkeit des Patienten in einer randomisierten kontrollierten Studie untersucht [11]. Die eingeschlossenen 49 Patienten wurden alle im Bereich des Unterschenkels mit Spalthaut transplantiert. Die eine Gruppe erhielt eine VS (28 Patienten) und wurde noch am selben Tag wieder entlassen, die zweite Gruppe (21 Patienten) bekam einen Überknüpfungsverband und wurde klassischerweise 5 Tage stationär immobilisiert. Die ambulante Versor- 

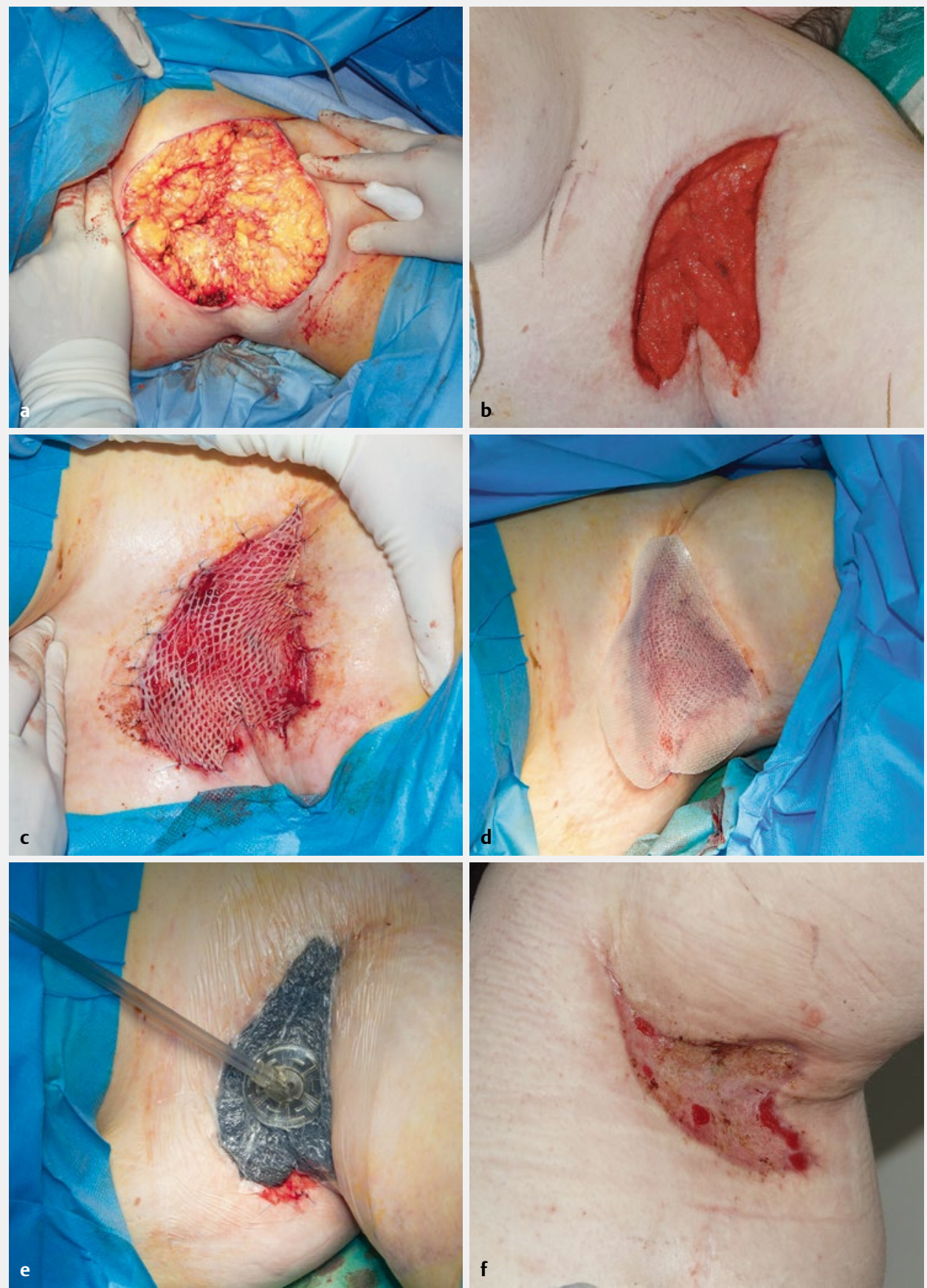

-Abb. 2 Vakuumversiegelung eines Spalthauttransplantats in asymmetrischer und beweglicher Region nach Exzision einer axillären Hidradenitis suppurativa. a Exzisionsdefekt axillär links; b Zustand nach 7 Tagen Vakuumversiegelung vor Transplantation; c mit einigen Fixationsnähten befestigtes Spalthauttransplantat; $\mathbf{d}$ Silikondistanzgitter zwischen Transplantat und Schwamm; e Vakuumversiegelung über dem Transplantat; f Zustand nach 5 Tagen Vakuumversiegelung.

gung der Patienten mit der VS führte im Durchschnitt zu einer Halbierung der Kosten bei gleichem klinischen Ergebnis.

\section{Retrospektive Fallserien}

Neben den randomisiert kontrollierten Studien finden sich in der Literatur auch noch eine Vielzahl von retrospektiven Fallserien un- terschiedlicher Qualität. Die wichtigsten mit mehr als 20 Patienten seien hier erwähnt.

Die größte Studie ist von Blume et al. mit insgesamt $142 \mathrm{~Pa}$ tienten nach Operationen im Bereich des Fußes und Sprunggelenkes [21]. 87 Patienten fanden sich in der VS-Gruppe und 55 in der Kontrollgruppe mit klassischem Überknüpfungsverband. Es zeigte sich eine signifikant bessere Einheilungsrate bei VS von $96 \%$ ver- 
sus $83 \%$ in der Kontrollgruppe. Zusätzlich traten unter VS wesentlich weniger Komplikationen wie Serome, Hämatome oder Wundinfektionen auf (3\% versus 16\%). Die Hospitalisationszeiten der Patienten in beiden Gruppen zeigten hingegen keinen signifikanten Unterschied.

Die retrospektive Studie von Körber et al. untersuchte 74 meshgrafts bei 54 Patienten mit chronischen Unterschenkelulzera [13]. 28 der Transplantate wurden mit VS versorgt, 46 mit klassischer Verbandstechnik. Die Einheilungsrate lag bei den VS Patienten bei $93 \%$ und in der Kontrollgruppe bei $67 \%$. Diese Ergebnisse geben einen Hinweis darauf, dass insbesondere bei chronischen schlecht heilenden Wunden die Vakuumversiegelung der klassischen Verbandstechnik überlegen zu sein scheint und damit gerade für Problemwunden besonders geeignet ist.

Carson et al. untersuchten 50 Patienten mit chronischen Wunden unterschiedlicher Ursache [23]. Eine Kontrollgruppe wurde nicht ausgewertet. Alle Patienten wurden vor der Spalthauttransplantation mittels VS zur Granulationsinduktion vorbehandelt, dann transplantiert und postoperativ mit VS versorgt. Alle Transplantate heilten komplett ein.

Scherer et al. untersuchte 34 Patienten mit Spalthauttransplantation und postoperativer VS bei verschiedenen Defektarten [21]. Als Kontrollgruppe wurden 27 Patienten mit Überknüpfungsverband herangezogen. Auch in dieser Studie zeigte sich eine Überlegenheit der VS. So musste bei 3 \% der Patienten mit VS eine erneute Transplantation durchgeführt werden, wohingegen bei $19 \%$ der Kontrollgruppe eine erneute Operation notwendig wurde. Keine Unterschiede zeigten sich bei der Länge des Aufenthalts in der Klinik oder der prozentualen Fläche der Einheilung zwischen den beiden Gruppen.

\section{Studien zur Vakuumversiegelung in Kombination mit Dermisersatz und Spalthauttransplantation}

Bei freiliegenden Sehnen, sehr tiefen Defekten oder auch Lokalisationen, bei denen eine zu große Kontraktion befürchtet wird, kommen immer häufiger Dermisersatzpräparate (z. B. Integra ${ }^{\circledR}$ oder AlloDerm ${ }^{\circledR}$ ) zur Anwendung. Um eine schnelle Deckung zu ermöglichen, werden häufig Dermisersatz und Spalthaut zusammen in einer Operation transplantiert.

Kim et al. untersuchten in einer prospektiv kontrollierten Studie, ob sich die Einheilungsraten, die Zeit bis zur kompletten Heilung und die Anzahl der notwendigen Verbandswechsel in der VS-Gruppe verbessern [25]. Insgesamt wurden 47 Patienten untersucht. 37 Patienten in der VS-Gruppe und 10 Patienten in der Kontrollgruppe. Nach 5 Tagen kam es in der VS-Gruppe zu einer Einheilungsrate von $98 \%$ in der Kontrollgruppe zu $84 \%$. Die komplette Heilung konnte man nach im Mittel 5,8 Tagen bei den VS-Patienten und nach 8,9 Tagen bei den Kontrollpatienten beobachten. Bei der VS war bis zur kompletten Heilung außer der Abnahme des Verbandes keine extra Wechsel notwendig, in der Kontrollgruppe im Mittel drei Verbandswechsel. Diese Studie ist die erste die zeigen kann, dass eine Kombination aus Dermisersatz, Spalthauttransplantat und Vakuumversiegelung nicht nur möglich ist, sondern auch zu sehr guten Einheilungsraten führt.

\section{Fazit}

Insgesamt ist die Evidenzlage für die Verwendung der postoperativen Vakuumversiegelung nach Spalthauttransplantation noch sehr eingeschränkt, auch wenn eine kürzlich erschienen Metaanalyse der verfügbaren Daten von Yin et al. zeigt, dass die Einheilungsraten signifikant verbessert und es auch zu weniger Re-Operationen kommt [26]. Trotz allem bedarf es noch größerer und gut gemachter randomisiert prospektiv kontrollierter Studien, um eine endgültige Aussage treffen zu können und die im stationären Setting definitiv höheren Kosten zu rechtfertigen. Somit ist sicher nicht jede unkomplizierte Spalthauttransplantation bei gut durchblutetem Wundgrund mit einer Vakuumversiegelung zu versorgen. Bei chronischen Wunden, schlechtem Wundgrund, hämodynamisch eingeschränkten lokalen Faktoren sowie in Bereichen von größerer Beweglichkeit ist eine postoperative Vakuumversiegelung nach Spalthauttransplantation definitiv in Erwägung zu ziehen. Auch die wesentlich bessere Mobilisierbarkeit der Patienten kann einen großen Vorteil für die Allgemeinsituation des Patienten bieten und zusätzlich das Thromboserisiko senken.

\section{Interessenkonflikt}

Der Autor gibt an, dass kein Interessenkonflikt besteht.

\section{Literatur}

[1] Argenta LC, Morykwas MJ. Vacuum-assisted closure: A new method for wound control and treatment: Clinical experience. Ann Plast Surg 1997; 38: 563-577

[2] Blume PA, Key J], Thakor P et al. Retrospective evaluation of clinical outcomes in subjects with split-thickness skin graft: Comparing V. A.C. ${ }^{\circledR}$ therapy and conventional therapy in foot and ankle reconstructive surgeries. Int Wound J 2010; 7: 480-487

[3] Carson SN, Overall K, Lee-Jahshan S et al. Vacuum-assisted closure used for healing chronic wounds and skin grafts in the lower extremities. Ostomy Wound Manage 2004; 50: 52-58

[4] Chen D, Zhao Y, Li Z et al. Circulating fibrocyte mobilization in negative pressure wound therapy. J Cell Mol Med 2017; 21: 1513-1522

[5] Chin MS, Ogawa R, Lancerotto $L$ et al. In vivo acceleration of skin growth using a servo-controlled stretching device. Tissue Eng Part $C$ Methods 2010; 16: 397-405

[6] Dainty LA, Bosco JJ, McBroom JW et al. Novel techniques to improve split-thickness skin graft viability during vulvo-vaginal reconstruction. Gynecol Oncol 2005; 97: 949-952

[7] Guoqi W, Zhirui L, Song W et al. Negative pressure wound therapy reduces the motility of Pseudomonas aeruginosa and enhances wound healing in a rabbit ear biofilm infection model. Antonie Van Leeuwenhoek 2018; 111: 1557-1570

[8] Hsiao SF-Y, Ma H, Wang Y-H et al. Occlusive drainage system for splitthickness skin graft: A prospective randomized controlled trial. Burns 2017; 43: 379-387

[9] Hsu C-C, Chow S-E, Chen CP-C et al. Negative pressure accelerated monolayer keratinocyte healing involves $\mathrm{Cdc} 42$ mediated cell podia formation. J Dermatol Sci 2013; 70: 196-203

[10] Kairinos N, Solomons M, Hudson DA. The paradox of negative pressure wound therapy - in vitro studies. J Plast Reconstr Aesthet Surg 2010; 63: $174-179$ 
[11] Ker H, Al-Murrani A, Rolfe G et al. WOUND Study: A Cost-Utility Analysis of Negative Pressure Wound Therapy After Split-Skin Grafting for Lower Limb Skin Cancer. J Surg Res 2019; 235: 308-314

[12] Kim EK, Hong JP. Efficacy of negative pressure therapy to enhance take of 1-stage allodermis and a split-thickness graft. Ann Plast Surg 2007; 58: $536-540$

[13] Korber A, Franckson T, Grabbe S et al. Vacuum assisted closure device improves the take of mesh grafts in chronic leg ulcer patients. Dermatology 2008; 216: 250-256

[14] Li T, Wang G, Yin P et al. Effect of negative pressure on growth, secretion and biofilm formation of Staphylococcus aureus. Antonie Van Leeuwenhoek 2015; 108: 907-917

[15] Llanos S, Danilla S, Barraza C et al. Effectiveness of negative pressure closure in the integration of split thickness skin grafts: a randomized, double-masked, controlled trial. Ann Surg 2006; 244: 700-705

[16] Lu F, Ogawa R, Nguyen DT et al. Microdeformation of three-dimensional cultured fibroblasts induces gene expression and morphological changes. Ann Plast Surg 2011; 66: 296-300

[17] Messerschmidt A, Meissner M, Kaufmann R et al. Local treatment of chronic wounds. Phlebologie 2016; 45 (2): 106-112

[18] Moisidis E, Heath T, Boorer C et al. A prospective, blinded, randomized, controlled clinical trial of topical negative pressure use in skin grafting. Plast Reconstr Surg 2004; 114: 917-922
[19] Morykwas MJ, Simpson J, Punger K et al. Vacuum-assisted closure: State of basic research and physiologic foundation. Plast Reconstr Surg 2006; 117(7 Suppl): 121S-126S

[20] Petkar KS, Dhanraj P, Kingsly PM et al. A prospective randomized controlled trial comparing negative pressure dressing and conventional dressing methods on split-thickness skin grafts in burned patients. Burns 2011; 37: 925-929

[21] Scherer LA, Shiver S, Chang M et al. The vacuum assisted closure device: a method of securing skin grafts and improving graft survival. Arch Surg 2002; 137 (8): 930-933

[22] Schneider AM, Morykwas M], Argenta LC. A new and reliable method of securing skin grafts to the difficult recipient bed. Plast Reconstr Surg 1998; 102: 1195-1198

[23] Valesky EM, Kaufmann R, Meissner M. Special indications for negative pressure wound therapy in dermatologic surgery. Hautarzt 2013; 64 (8): 585-591

[24] Wackenfors A, Sjogren J, Gustafsson R et al. Effects of vacuum-assisted closure therapy on inguinal wound edge microvascular blood flow. Wound Repair Regen 2004; 12: 600-606

[25] Weinfeld AB, Kelley P, Yuksel E et al. Circumferential negative-pressure dressing (VAC) to bolster skin grafts in the reconstruction of the penile shaft and scrotum. Ann Plast Surg 2005; 54: 178-183

[26] Yin Y, Zhang R, Li S et al. Negative-pressure therapy versus conventional therapy on split-thickness skin graft: A systematic review and meta-analysis. Int J Surg 2018; 50: 43-48 